Rev Biomed 2003; 14:35-43.

\title{
La enfermedad de Chagas en el Estado de Yucatán, México. (1940-2002).
}

\section{Contribuciones de la UADY \\ a la Medicina}

Jorge E. Zavala-Velázquez.

Facultad de Medicina, Universidad Autónoma de Yucatán, Mérida, Yucatán, México.

\section{RESUMEN.}

La primera evidencia de enfermedad de Chagas en Yucatán surge en 1940, pero es hasta 1972 que se lleva al cabo la primera investigación formal sobre esta enfermedad. Este estudio demostró una prevalencia serológica de $11.2 \%$, encontrando también $12(0.5 \%)$ casos clínicos. En este mismo estudio se encontró que los vectores (triatoma) tenían una prevalencia de infección para T. cruzi del 17.3\%. Diecinueve años después la prevalencia en vectores seguía siendo similar (16\%), pero por primera vez se detecta un elevado índice de colonización y un año después se demuestra que entre los hábitos biológicos del triatoma el humano ocupa ya el primer lugar en preferencias cuando quince año antes ocupaba el cuarto lugar. En 1985 otro estudio demostró que la seroprevalencia se había incrementado a un $18 \%$. En la década de los 90's dos realizados en donadores de sangre demostraron la posibilidad de riesgo de contagio a través de la transfusión sanguínea. Estudios realizados en fechas recientes han permitido conocer que la temperatura tiene un papel preponderante en la capacidad transmisora del triatoma y que las cepas encontradas en Yucatán son filogenéticamente diferentes a las del resto del país. También se ha completado el panorama de los componentes proteicos mitocondriales del T. cruzi con lo que se tienen posibilidades de identificar blancos potenciales para la investigación de drogas terapéuticas. Con este trabajo se demuestra como el conocimiento alcanzado a lo largo del tiempo puede contribuir a la solución de problemas de salud que afectan a una población y como la investigación permite el desarrollo de nuevos conocimientos. (Rev Biomed 2003; 14:35-43)

Palabras clave: Enfermedad de Chagas, Tripanosiomiasis americana, Trypanosoma cruzi, Yucatán México.

\section{SUMMARY.}

Chagas' disease in the state of Yucatan, Mexico (1940-2002).

The first evidence of Chagas' disease in Yucatan appeared in 1940, but it was not until 1972 that the first investigation was carried out on this disease. This study demonstrated a serological prevalence of $11.2 \%$, and found 12 clinical cases. In this study the vectors (triatoma) found had a prevalence of infection for $T$.

Solicitud de sobretiros: Dr. Jorge Zavala-Velázquez. Facultad de Medicina, Universidad Autónoma de Yucatán. Avenida Itzáes No. 498 x 59 y 59-A, C.P. 97000, Mérida, Yucatán, México. Teléfono: (999) 92853 29; Fax: (999) 9240554

Recibido el 31/Octubre/2002. Aceptado para publicación el 18/Febrero/2003. 


\section{JE Zavala-Velázquez.}

cruzi of $17.3 \%$. Nineteen years later the prevalence in vectors continued to be similar (16\%), but for the first time a high index of colonisation was detected and a year later it was shown that among the biological habits of triatoma the human had moved to first place from the fourth place it had occupied fifteen years earlier. In 1985, another study showed that the seroprevalence had increased to $18 \%$.

During the 1990s two studies carried out on blood donors showed the possibility of blood transfusions being a risk of infection. Studies carried out recently have allowed us to determine that temperature has a preponderant role in the transmission capacity of the triatoma and that the strains found in Yucatan are philogenetically different to those from the rest of the country. The panorama of the protean mitochondrial componantes of T. cruzi has been completed giving posibilities of identifying potential targets for the investigation into therapeutic drugs. In this study, it is demonstrated how knowledge achieved over time can contribute to the solution of health problems affecting a population and how investigation allows for the development of new knowledge. (Rev Biomed 2003; 14:35-43)

Key words: Chagas' disease, Tripanosomiasis americana, Trypanosoma cruzi, Yucatan Mexico.

\section{INTRODUCCIÓN.}

La "Enfermedad de Chagas" denominada también Tripanosomiasis americana, se caracteriza por ser una infección generalizada que cursa clínicamente a partir de una forma aguda hasta llegar a la cronicidad, con un periodo asintomático que puede durar varios años y que se denomina forma indeterminada. Tiene como agente causal al protozoario flagelado Trypanosoma cruzi el cual es transmitido a los mamíferos incluyendo al hombre por insectos hematófagos de la familia Reduviidae.

Las primeras evidencias de la existencia en el Estado de Yucatán del parásito y de su existencia en vectores surge en 1940 con los informes de Eugenio Palomo y Luis Mazzotti (1,2). No es sino hasta 1964 cuando Biagi y Tay notifican de nuevo la existencia de T. cruzi en nuestro medio al encontrarlo en un pequeño número de triatomas capturadas en Chichen Itzá, Mérida y Tinún (3). En 1969 los Servicios Coordinados de Salud Publica del Estado le solicitan al recién creado laboratorio de "Investigación y pruebas especiales" dependiente de la Facultad de Medicina de la Universidad de Yucatán y del Hospital O'Horán la identificación de un parásito diferente a Plasmodiun observado por los microscopistas de la Campaña Nacional para la erradicación del Paludismo, en un paciente febril internado en el servicio de Pediatría del Hospital "20 de Noviembre", de la ciudad de Mérida, Yucatán, siendo dicho parásito un tripomastigote de T. cruzi. En el servicio de Medicina de mujeres del mismo hospital, se encontraba otra paciente con diagnóstico de posible fiebre tifoidea con conjuntivitis unilateral agregada, para la cual también se solicitó interconsulta porque las pruebas diagnosticas de confirmación resultaron negativas. La conjuntivitis resulto ser un clásico "signo de Romaña" y en el estudio directo y en el frotis de sangre se encontró también $T$. cruzi. Un dato de interés epidemiológico fue que los dos pacientes radicaban en Itzincab Palomeque, una pequeña hacienda ubicada a pocos kilómetros de la Ciudad de Mérida y que resultaron ser madre e hijo. En 1971 el Departamento de Patología Tropical en colaboración con el servicio de Cardiología del Hospital O'Horán, detectaron cuatro casos de cardiopatía chagásica, falleciendo uno de ellos el cual fue autopsiado encontrándose después de varios cortes histológicos un nido de amastigotes de T. cruzi.

\section{LA INVESTIGACIÓN FORMAL DEL PROBLEMA.}

A pesar de toda la información antes mencionada, y probablemente contagiados por el escepticismo que prevalecía a nivel nacional, no tan sólo sobre la importancia que esta enfermedad pudiera tener en la salud de la población mexicana, sino incluso en su existencia real en territorio nacional, que sostenían con terquedad altos funcionarios del Sector Salud, en el cuerpo médico local en su gran mayoría, así como en algunas autoridades gubernamentales,

\section{Revista Biomédica}




\section{La enfermedad de Chagas en Yucatán, México.}

también prevalecía la duda. Con el fin de informar a las autoridades competentes la importancia que esta antropozoonosis pudiera representar para la salud de la población de nuestro Estado, el Departamento de Patología Tropical de la Facultad de Medicina de la Universidad deYucatán, realizó en 1972, una investigación patrocinada por el Consejo Nacional de Ciencia y Tecnología (CONACYT-P027) y con el apoyo de Gobierno del Estado por medio de los Servicios Coordinados de Salud y de la Delegación del Instituto Mexicano del Seguro Social (IMSS).

El diseño de esta primera investigación formal sobre la Enfermedad de Chagas en Yucatán fue integral abordando los siguientes aspectos:

a).- Estudio serológico en población abierta para establecer la frecuencia de contacto de la población humana con el parásito.

b).- Establecer la frecuencia de la Enfermedad de Chagas mediante la detección de casos clínicos tanto en la fase aguda como crónica.

c).- Determinar cuál o cuáles son los géneros y las especies de vectores predominantes en el medio, así como su frecuencia de infección, período estacional de mayor densidad, preferencias de ecotopo (domiciliar-silvestre) y hábitos de alimentación.

d).- Determinar las especies de mamíferos inferiores que por sus hábitos y frecuencia de infección estuvieran participando como eslabón en la historia natural de la enfermedad.

c).- Frecuencia de casas cuyas características de construcción las hicieran propicias para la colonización por triatominos así como frecuencia con que los moradores habían sido picados por los insectos.

Para realizar los estudios seroepidemiológico se utilizaron las técnicas de hemoaglutinación indirecta con antígeno proporcionado por el CDC de Atlanta, E.E.U.U. y aglutinación con antígeno adsorto a partículas de látex de marca comercial. Los casos clínicos se detectaron a través de un equipo formado por un cardiólogo, un internista, un pediatra, un oftalmólogo, un patólogo y tres pasantes de medicina, así como con la colaboración de los servicios respectivos del Hospital O'Horán y del IMSS, las técnicas de diagnostico empleadas fueron las convencionales para las formas aguda y crónica. La captura de insectos en los domicilios se realizó en forma manual y los silvestres por medio de trampas de luz, la clasificación taxonómica de los insectos capturados se realizó en el Departamento y su confirmación en el Departamento de Parasitología de la Fac. de Medicina de la UNAM. La captura se realizó durante un año y la determinación de hábitos alimenticios por medio de la detección de sangre en las heces del insecto utilizado contra inmunoelectroforesis. Para definir las posibilidades de colonización por triatominos de las casas, éstas fueron clasificadas según su tipo de construcción en cuatro niveles de riesgo, siendo el grado I el de mayores posibilidades y siguiendo hasta el grado IV que representaba casi nulas probabilidades; con un sistema similar se clasificaron los patios de las casas, según hubiera en ellos sitios adecuados para el establecimiento de nidos de triatominos, así como de la existencia de animales y su cercanía con la casa habitación, quedando formados cinco niveles de riesgo.

Esta investigación se realizó en dos años obteniéndose los resultados siguientes:

Estudio seroepidemiológico.- Se dividió el Estado en tres zonas arbitrarias, las cuales se delimitaron por diferencias de situación geográfica y actividad agropecuaria clasificándolas como zona "A" localizada al noroeste y con predominio al cultivo del henequén y en la cual por ser la de mayor concentración de población se muestrearon 7 localidades: Motul, Hunucmá, Halachó, Dzemul, Kopomá, Chabihau e Itzincab. La zona "B" localizada al noreste y con predominio de la actividad ganadera y explotación de madera, en ella se muestrearon 3 localidades: Tizimín, Tinún y Pocopoch y la zona "C" localizada al sur y con predominio de cultivo de maíz y frutales, muestreándose 3 localidades: Peto, Tzucacab y Ticum. Todas las localidades muestreadas se seleccionaron en forma aleatoria. Se muestrearon 2,245 habitantes (sólo se tomó muestra a sujetos que dieron su anuencia), de ellos resultaron positivos al titulo de corte (1:16) 252 lo que dio una seroprevalencia del $11.22 \%$, 


\section{JE Zavala-Velázquez.}

siendo importante señalar que los positivos quedaron comprendidos en su mayoría entre los 5 y 54 años de edad, así como que la mayoría provenían de la zona "A".

Detección de casos clínicos.- Se detectaron 12 casos, siendo 7 en fase aguda y 5 en fase crónica. A tres pacientes en fase aguda se les trató con nitrofurano (Nifurtimox), desapareciendo la parasitemia a los 10 días así como la sintomatología, considerándolos como aparentemente curados. Es importante resaltar que esta fue la primera vez que se utilizó este medicamento en México y el cual fue obsequiado por la compañía farmacéutica productora, y que ahora es de uso común.

Estudio de transmisores.- Se capturaron y clasificaron 4,019 insectos vectores, todos ellos resultaron ser del género Triatoma y de la especie dimidiata. De ellas el 64\% fueron capturadas dentro de las casas y el 36\% en el área selvática. Solo pudieron ser estudiadas 1579 para la búsqueda de infección natural, de ellas 296 demostraron estar infectadas por tripomastigotes del género Trypanosoma, pero solamente 274 (17.3\%) resultaron ser $T$. cruzi, probablemente el número restante lo estaba con $T$. rangeli. El mayor número de insectos fue capturado durante los meses de agosto a noviembre. Así mismo la mayor captura se realizó en la zona " $A$ " (henequenera). La mayor preferencia alimenticia del triatoma fue hacia la zarigüeya (43\%), siguiéndole en importancia el armadillo $(25 \%)$ y el perro (19\%), un dato curioso es que no se encontró antropofilia, sin embargo se llegó a la conclusión de defectos en el suero antihumano utilizado y que por lo tanto debería ser reanalizado, ya que era incongruente con los resultados de prevalencia y con los casos reportados.

Estudio de hábitat humano.- Se estudiaron 1,137 casas de las cuales el $60 \%$ estaban comprendidas entre los altos niveles de riesgo (I y II) de colonización por triatominos, que el $46 \%$ tenían anexos (patios) con niveles de riesgo altos (I a III), el 90\% convivían con animales tanto domésticos como sinantrópicos y el $21 \%$ habían sido picados por triatomas, siendo un hecho importante el que todos ellos lo hubieran sufrido dentro de sus casas $(4,5)$. Los resultados obtenidos demostraron sin lugar a dudas la existencia en el Estado de los tres eslabones que constituyen la historia natural de la Tripanosomiasis americana, que existía un entrelace de ellos que daban como resultado final que se considerara a Yucatán como una zona endémica de la misma y que podría ser de importancia dentro del esquema epidemiológico estatal, situación que podría esclarecerse por medio de estudios más profundos y precisos de cada uno de los elementos que constituyen el sostén de la enfermedad: el transmisor, los reservorios y el hombre mismo. Las investigaciones sobre los aspectos antes mencionados, lo ha venido realizando la Universidad Autónoma de Yucatán hasta la fecha, por medio de investigadores, principalmente del Centro de Investigaciones Regionales, los cuales han logrado aportar importante información que se detalla cronológicamente a continuación.

\section{ENFOQUES Y RESULTADOS POSTERIORES.}

El interés que despertó este primer estudio y la necesidad de profundizar más en algunos aspectos, así como resolver algunas interrogantes que dejara, originó que se siguieran realizando importantes investigaciones en los tres aspectos fundamentales que conforman la historia natural de la enfermedad de Chagas. Los enfoques y sus resultados se presentan a continuación en orden cronológico.

\section{TRANSMISORES.}

Quintal R y Polanco G, en 1977 repiten el estudio de preferencias alimenticias de $T$. dimidiata, utilizando para ello la misma técnica de contra inmunoelectroforesis, pero con antisueros más específicos y purificados, señalando por primera vez la antropofilia del insecto, aunque ésta ocupa un cuarto lugar, siendo la gallina y la zarigüeya las dos primeras (6). En 1991 Guzmán-Marín y col. realizan un estudio en 116 viviendas en 22 localidades de 7 municipios de Estado, con el fin de determinar índices

\section{Revista Biomédica}




\section{La enfermedad de Chagas en Yucatán, México.}

entomológicos de T. dimidiata, con los resultados siguientes: se capturaron 269 triatomas de los cuales el $16 \%$ estaba infectado con T. cruzi, un índice de dispersión de $95 \%$, infestación de $61 \%$, colonización $25 \%$, densidad $231 \%$ y concentración $378 \%$ (7). Como puede observarse la frecuencia de infección por el parásito en los vectores permanecía estable después de 17 años así como también, la presencia de los mismos en las casas habitación (infestación), pero por primera vez se demuestra un índice elevado de colonización, es decir de capacidad de $T$. dimidiata para establecer nichos de reproducción dentro de las mismas. En 1992 Guzmán-Marín y col. por medio de un diseño experimental, analiza aspectos biológicos intrínsecos del insecto como son: tiempo de repleción, de defecación, cantidad de sangre que ingiere así como el tiempo que transcurre del huevo hasta llegar al estado adulto. Para completar el análisis de los hábitos biológicos se vuelven a estudiar las preferencias alimenticias de triatomas capturados en distintas localidades de siete municipios del Estado, encontrando que a 15 años del estudio de Quintal y Polanco el humano ocupaba ya el primer lugar en sus preferencias, seguido de aves, perro y zarigüeya entre otras especies estudiadas. Con estos resultados los autores concluyen que $T$. dimidiata se comporta como un buen vector de T. cruzi en el Estado (8).

Es importante señalar que los altos índices de infestación y de establecer nicho ecológico domiciliar (colonización), así como la marcada preferencia alimenticia de $T$. dimidiata hacia el hombre y el perro, animal con el que convive íntimamente, sobre todo en el área rural, como lo demostraron las investigaciones antes mencionadas, se establece en nuestro Estado una expedita dinámica de trasmisión intradomiciliar. Si comparamos el índice de riesgo de transmisión (46\%),medido por el porcentaje de viviendas en donde existía esa posibilidad con el reportado en la investigación de 1972 (60\%), es evidente que en 20 años solo hubo una relativa mejoría en el tipo de construcción de la casas en el área rural del Estado, de persistir esta situación en la actualidad, sería un aspecto importante a considerar para el buen éxito de cualquier programa de control que se desee implantar.

En 1994 Guzmán-Marín y col. analizan la temperatura como un factor de diferenciación hasta la forma infectante de $T$. cruzi en el triatoma, concluyendo que la temperatura promedio reinante en el Estado $\left(28^{\circ} \mathrm{C}\right)$ favorece la capacidad de Triatoma dimidiata, como transmisor en la región (9). El grupo de investigación encabezado por Eric Dumontiel realizó en 1999,un estudio sobre la distribución de Triatoma dimidiata y la dinámica de transmisión por T. cruzi en la Península de Yucatán, reportando que hay baja colonización pero alta infestación, concentra en el norte de la Península principalmente en los meses calurosos y secos de abril a junio y también que las casas estaban infestadas por adultos machos con capacidad de volar, por lo que sugieren que este hecho es parte importante de la dinámica de transmisión en la región (10).

\section{ESTUDIOS EN POBLACIÓN HUMANA.}

Seroepidemiología.- Farfán-Ale y col. en 1985 realizan un estudio seroepidemiológico en una muestra representativa de la población en la búsqueda de anticuerpos contra $T$. cruzi y $T$. gondii, utilizando como técnica la inmunofluorescencia, reportando una prevalencia para el primero del $18 \%$, siendo ya superior en un $2 \%$ al reportado en el primer estudio realizado en Yucatán (11).

Casos clínicos.- Zavala-Velázquez y col. en 1975 reportan la existencia de 4 casos en fase aguda, tres del sexo femenino con edades de 4, 15 y 25 años y uno masculino de 18 meses de edad. Tres de ellos fueron tratados con nifurtimox y uno con metronidazol, todos con evolución satisfactoria y cura parasitológica (12). Quintal y col. informan de una revisión de los casos habidos en Yucatán durante tres años (1972 a 1975), según su curso clínico, resultado 6 casos en fase aguda y 5 en fase crónica (13). En 1990 BarreraPérez y col. reportan una revisión de los casos en fase aguda de 1970 a 1989, analizándolos desde el punto de vista de su evolución clínica y pronóstico en dos categorías: leve y benigna aquellos en los que la enfermedad no pone en peligro la vida del paciente e 


\section{JE Zavala-Velázquez.}

intensa y grave aquellos en los que estuvieron comprometidos órganos o sistemas que de alguna manera pusieran en peligro la vida del paciente. Se analizaron 24 pacientes de los cuales 14(58.3\%) cursaron con una evolución intensa y pronóstico grave y $10(41.6 \%)$ con evolución leve y pronóstico benigno (14). Este hecho planteó la necesidad de estudiar más a fondo al parásito en cuanto a su virulencia, ya que el estudio inicial (1972-74) sugería la posible baja virulencia de la cepa yucateca. Otro hecho interesante de este estudio es que las dos terceras partes de los enfermos fueron del sexo femenino y una cuarta parte correspondió al grupo etario entre 0 y 15 años, ya que por un lado reafirma la domiciliaridad de los triatomas y por otro demuestra que la transmisión permanecía activa desde el momento en que se inició la investigación de la enfermedad de Chagas en el Estado hasta el momento del corte.

Barrera-Pérez y col. en 1992 realizan un estudio de prevalencia de la enfermedad. Para ello estudiaron a todos los miembros de 86 familias de 7 municipios, encontrando 6 casos $(1.44 \%)$ de 416 personas estudiadas. De ellos uno correspondió a la forma crónica asintomática (indeterminada) y cinco en la fase crónica sintomática. Todos los casos vivían en el área rural. La paciente crónica asintomático fue tratada con nifurtimox y al término del tratamiento quedo negativa parasitológicamente y con disminución del título serológico. A parte del dato de prevalencia, este estudio reporta por primera vez la forma "indeterminada" en el medio, así mismo es importante señalar que dada la corta edad (14 años) del paciente y el hecho de que estuviera en fase crónica sintomática, sugiere fuertemente que la infección pudo haberse realizado "in útero" (15). En 1995 Zavala-Castro y col. reportan cardiopatía chagásica en 7 de 36 pacientes del servicio de cardiología de Hospital O'Horán de Mérida, Yucatán. Sin embargo de ellos sólo 5 (14\%) eran originarios de Yucatán, todos presentaron cardiomegalia grado III y uno falleció por lo que fue autopsiado, encontrándose 2 nidos de amastigotes en el estudio histopatológico del corazón. Este es el primer estudio de frecuencia de la forma crónica de la enfermedad en la región (16).
Riesgo de transmisión a través de donadores de sangre.- En 1991, Barrera-Pérez y col realizan un estudio piloto con 96 donadores del banco de sangre de hospital O'Horán con la técnica de hemoaglutinación indirecta, encontrando el $6.12 \%$ de positivos (17). Rodríguez-Félix y col en 1995, amplían el estudio en donadores del mismo banco utilizando para ello la detección de $\operatorname{IgG}$ e IgM con la técnica de inmunofluorescencia, encontrando el $5.6 \%$ de positivos de 215 estudiados concluyendo que el riesgo de transmisión, aplicando la formula de Cerisola, fluctúa entre $5.6 \%$ y $68.3 \%$ dependiendo del número de transfusiones recibidas, que en el hospital antes mencionado varió de 1 a 20 por paciente (18). Estos dos estudios vinieron a demostrar que el riesgo de transmisión vertical (hombre-hombre) en nuestro medio, era importante de tomarse en consideración. Esta situación en la actualidad esta controlada por la norma que rige para los bancos de sangre que operan oficialmente en el País.

\section{ESTUDIOS SOBRE CARACTERÍSTICAS DEL PARÁSITO.}

Zavala-Castro y col. en 1992, analizan por primera vez el perfil molecular de las cepas yucatecas y demuestran su separación filogenética de las del resto del País, a sí mismo, que el comportamiento biológico de ellas que aparentemente es diferente a las demás, tiene una posible explicación en su origen y evolución (19). En 1995 el mismo autor con otros colaboradores, estudian aspectos relacionados con el fenómeno de diferenciación del parásito, llegando a la conclusión de que el aumento de cAMP intracelular inducido por ligantes adrenérgicos o toxina del cólera en células infectadas con T. cruzi, es capaz de inducir dicho proceso (20). En el 2000, Zavala-Castro y col. reportan la existencia de proteínas que se unen al kinetoplasto en los estadíos de amastigote y tripomastigote de $T$. cruzi y proponen tres clases principales de estas proteínas, además sugieren su posible función en la organización del ADN mitocondrial (21). Arankowsky-Sandoval y col. en el 2001 investigan en un modelo experimental con ratas, alteraciones funcionales que pudiera ocasionar

\section{Revista Biomédica}




\section{La enfermedad de Chagas en Yucatán, México.}

la infección por T. cruzi al sistema nervioso central encontrando deficiencias tanto en el sueño como en la memoria y sugieren que la rata es un modelo adecuado para estudios sobre disfunción cerebral en la enfermedad de Chagas (22). En ese mismo año Barrera-Pérez y col, analizan el comportamiento biológico de tres cepas de $T$. cruzi aisladas de humanos en Yucatán, dos de ellas en pacientes con patología severa y una en un paciente asintomático (fase indeterminada). Los autores concluyen que estas cepas tienen una virulencia comparable con las de otras regiones, pero que habría que relacionarlo posteriormente con sus marcadores genéticos (23). Zavala-Castro y col en el 2002, describen el perfil proteico asociado al kinetoplasto de la forma de epimastigote de $T$. cruzi. Con estos datos completan el panorama de los componentes proteicos mitocondriales del parásito con lo que se abre la posibilidad de identificar blancos potenciales para la investigación de drogas terapéuticas (24).

\section{ESTUDIOS SOBRE RESERVORIOS.}

En 1989 Zavala-Velázquez y col. para determinar la infección natural por $T$. cruzi en mamíferos del Estado, analizaron 372 animales selváticos y sinantrópicos, que incluían carnívoros, marsupiales y roedores por medio de la detección de anticuerpos con la técnica de hemoaglutinación así como búsqueda del parásito utilizando métodos directos (observación de sangre en fresco, frotis, inoculación a ratón e histopatología). Los resultados demostraron una frecuencia de positividad del $18.54 \%$, siendo esta significativamente mayor en los selváticos que en los sinantrópicos, y concluyen que por la positividad serológica y parasitológica encontrada así como por su comportamiento en el medio ambiente, aunado a características socioeconómicas y culturales de la población, Canis familiaris, Didelphys marsupialis y Rattus rattus, actúan como elementos de enlace entre el ciclo selvático y domiciliar (25). En 1996 Ruiz-Piña y CruzReyes, llevan a cabo un estudio para determinar el papel de Didelphys virginiana como reservorio sinantrópico en una localidad (Dzidzilché) de Yucatán y la estacionalidad de la transmisión de $T$. cruzi en la misma. Reportan el 53.9\% de positividad en 102 animales estudiados así como una mayor densidad de triatomas en el periodo comprendido de marzo a mayo. Por los resultados obtenidos los autores sugieren una estacionalidad en la transmisión peridoméstica de T. Cruzi (26).

\section{INVESTIGACIONES EN PROCESO EN EL 2002.}

En el Departamento de Patología Tropical de la Facultad de Medicina, el Dr. Víctor Suárez y colaboradores han finalizado un estudio sobre la frecuencia de cardiopatía chagásica crónica en el Estado, encontrando que en pacientes con diagnóstico de cardiopatía dilatada un $37 \%$ presentaron anticuerpos contra T. cruzi y en el $25 \%$ de los mismos, fue posible amplificar fragmentos del minicírculo del parásito. Este año iniciaron investigaciones sobre aspectos clínicos (fase aguda e indeterminada) y sobre eventos que ocurren durante el proceso fisiopatogénico de la enfermedad.

En el Centro de Investigaciones Regionales "Dr. Hideyo Noguchi”, en el laboratorio de Biología Celular, el Dr. Zavala-Castro y su grupo, pretenden caracterizar los componentes proteicos asociados con el ADN del kinetoplasto de T. cruzi, para determinar su localización y función dentro del genoma mitocondrial del parásito y de esta manera se identificarán cuáles son las moléculas asociadas con el mantenimiento de la estructura génica mitocondrial y cuáles son las moléculas esenciales para la vida del mismo, con el fin de probar drogas dirigidas a desestabilizar la función de la mitocondria y por ende que afecten la vida del parásito. Realizan estudios de clonación y caracterización de posibles receptores beta adrenérgicos en la superficie del parásito, los cuales permitirán un mejor conocimiento sobre los mecanismos fisiopatogénicos de la enfermedad. También han iniciado estudios para identificar compuestos naturales que tengan efectividad contra T. cruzi y otras parasitosis de importancia humana.

En el laboratorio de Parasitología, el Dr. Eric Dumontiel y sus colaboradores han empezado a 


\section{JE Zavala-Velázquez.}

caracterizar aspectos claves de la biología y ecología de $T$. dimidiata, como son su distribución geográfica, su estacionalidad y la dinámica de transmisión de $T$. cruzi a humanos, que han dado como resultado una primera estimación de las zonas y épocas de alto riesgo de transmisión. Están elaborando mapas predictivos detallados de distribución del vector y zonas de riesgo de transmisión, así como modelos de la dinámica de transmisión de $T$. cruzi y evalúan la eficacia de diferentes estrategias de control, basadas en las características biológicas de $T$. dimidiata. También realizan investigaciones sobre nuevas terapias y sobre vacunas de ADN, que puedan ser usadas para curar y prevenir la enfermedad de Chagas.

En el mismo laboratorio el Dr. Mario Barrera Pérez, trabaja en la evaluación de distintos productos quimioterapéuticos contra T. cruzi, en cooperación con un grupo de investigadores en parasitología, bioquímica y química inorgánica de la Facultad de Ciencias de la Universidad de Granada, España. También realiza investigaciones en epidemiología, específicamente en el aspecto de transmisión vectorial activa, así como en aspectos clínicos de la enfermedad de Chagas, en especial de la fase indeterminada, esto último en colaboración con el grupo del Departamento de Patología Tropical de la Facultad de Medicina.

El Dr. Hugo Ruiz Piña, igualmente del Laboratorio de Parasitología, trabaja en el estudio de los mecanismos inmunológicos de Didelphys virginiana contra cepas yucatecas de Trypanosoma cruzi y en el conocimiento de los aspectos básicos de la oviposición del principal vector de T. cruzi en varias poblaciones de la Península de Yucatán, para poder entender su influencia en la abundancia observada en la Península de Yucatán.

\section{COLOFÓN.}

Esta historia es un ejemplo entre muchos, del compromiso social que asumen las universidades públicas, en este caso la Autónoma de Yucatán, en el análisis y posible solución de problemas de salud que afectan a la población y que representan también una importante carga económica para el Estado. El conocimiento alcanzado a lo largo del tiempo y la tecnología con que cuentan los investigadores universitarios, considero debería ser aprovechada por las instituciones rectoras del área de salud tanto del país como de los estados, para diseñar el programa de control del caso que nos ocupa, la Enfermedad de Chagas.

\section{REFERENCIAS.}

1.- Mazzotti, L. Triatomineos de México y su infección natural por T. cruzi. Chagas Med (Mex) 1940; 20:95.

2.- Palomo-Erosa, E. Triatoma dimidiata (Lat) de Yucatán. Algunas consideraciones sobre su prevalencia, distribución, biología e infección natural por Trypanosoma cruzi. 1940.Tesis Fac. Ciencias Físico-Químicas. Universidad de Yucatán. México.

3.- Tay J, de Biagi AM. Localidades nuevas de triatominos mexicanos y su infección natural por Trypanosoma cruzi. Rev Fac Med Mex 1964; 6: 305-9.

4.- Zavala-Velázquez J. Enfermedad de Chagas en Yucatán (1972-74). Informe final del Proyecto 027.1974.CONACYT.

5.- Pinzón-Cantarell J, Quintal-Avilés R, Zavala-Velázquez, J. La enfermedad de Chagas en el Estado de Yucatán, México. (informe de transmisores). Salud Pública Mex 1976; 18: 9991003 .

6.- Quintal R, Polanco G. Feeding preferences of Triatoma dimidiata maculipenis in Yucatan, Mexico. Am J Trop Med Hyg 1977; 26:176-8.

7.- Guzmán-Marín ES, Barrera-Pérez MA, Rodríguez-Félix ME, Escobedo-Ortegón FJ, Zavala-Velázquez JE. Índices entomológicos de Triatoma dimidiata en el Estado de Yucatán. Rev Biomed 1991; 2: 20-9.

8.- Guzmán-Marín ES, Barrera-Pérez MA, Rodríguez-Félix ME, Zavala-Velázquez JE. Hábitos biológicos de Triatoma dimidiata en el Estado de Yucatán, México. Rev Biomed 1992; 3: 125-31.

9.- Guzmán-Marín ES, Barrera-Pérez MA, Rodríguez-Félix ME, García-Rejón JE. La temperatura como factor de diferenciación de Trypanosoma cruzi en Triatoma dimidiata. Rev Biomed 1994; 5: 33-7.

10.-Dumonteil E, Gourbiere S, Barrera-Pérez M, RodríguezFélix E, Ruiz-Piña H, Baños-López O, et al. Geographic distribution of Triatoma dimidiata and transmission dynamic of Trypanosoma cruzi in the Yucatan Peninsula of Mexico.

\section{Revista Biomédica}




\section{La enfermedad de Chagas en Yucatán, México.}

Am J Trop Med Hyg 2002, 67:176-83.

11.- Farfán-Ale JA, Loroño-Pino MA, Flores-Flores LF, Rosado-Paredes EP, Arjona-Torres AI. Prevalencia de anticuerpos contra Toxoplasma gondii y Trypanosoma cruzi en el Estado de Yucatán, México. Rev Biomed 1992; 3: 8-12.

12.- Zavala-Velázquez J, Rodríguez-López MH, Baqueiro-Díaz A. Enfermedad de Chagas en el Estado de Yucatán, México. Informe de cuatro casos clínicos. Patología 1975; 13: 355-63.

13.- Quintal-Avilés R, Zavala-Velázquez J, Rodríguez-López M. La enfermedad de Chagas en el Estado de Yucatán, México. Revisión clínica. Rev Invest Clin 1975; 27: 255-8.

14.- Barrera-Pérez MA, Rodríguez-Félix ME, Guzmán-Marín ES, Zavala-Velázquez JE. Enfermedad de Chagas en el Estado de Yucatán. Revisión de casos clínicos en fase aguda de 1970-1989. Rev Biomed 1990; 1: 185-5.

15.- Barrera-Pérez MA, Rodríguez-Félix ME, Guzmán-Marín ES, Zavala-Velázquez JE. Prevalencia de la Enfermedad de Chagas en el Estado de Yucatán. Rev Biomed 1992; 3: 133-9.

16.- Zavala-Castro JE, Gutiérrez-Flota H, Barrera-Pérez MA, Bolio-Solís AJ, Zavala-Velázquez JE. Cardiopatía chagásica crónica detectada en pacientes del hospital General Regional O Horán, Mérida, Yucatán, México. Arch Inst Cardiol Mex 1995; 65: 541-5.

17.- Barrera-Pérez MA, Guzmán-Marín ES, Rodríguez-Félix ME, Zavala-Velázquez JE. Estudio piloto para la detección de anticuerpos contra Trypanosoma cruzi en un grupo de donadores de sangre del Estado de Yucatán. Rev Biomed 1991;2: 15-9.

18.- Rodríguez-Félix ME, Zavala-Velázquez JE, Barrera-Pérez MA, Guzmán-Marín E, Ramírez-Sierra MJ, Álvarez-Moguel R. Riesgo de transmisión de la Enfermedad de Chagas por donantes de sangre. Rev Biomed 1995; 6: 70-5.

19.- Zavala-Castro JE, Velazco-Castrejón O, Hernández R. Molecular characterization of Mexican stocks of Trypanosoma cruzi using total DNA. Am J Trop Med Hyg 1992; 47: 201-9.

20.- Zavala-Castro JE, Guzmán-Marín E, Zavala-Velázquez JE. Adrenergic ligands trigger intracellular differentiation of Trypanosoma cruzi. Arch Med Res 1995; 26: 449-50.

21.- Zavala-Castro JE, Acosta-Viana K, Guzmán-Marín E, Rosado-Barrera ME, Rosales-Encina JL. Stage specific kinetoplast DNA-binding proteins in Trypanosoma cruzi. Acta Tropica 2000; 76: 139-46.

22.- Arankowsky-Sandoval G, Mut-Martín M, SolísRodríguez F, Góngora-Alfaro JL, Barrera-Pérez MA. Sleep and memory deficits in the rat produced by experimental infection with Trypanosoma cruzi. Neuroscience Letters 2001; 306: 65-8.

23.- Barrera-Pérez MA, Rodríguez-Félix ME, Guzmán-Marín E, Zavala-Velázquez JE, Dumonteil E. Biological behavior of three strains of Trypanosoma cruzi from Yucatan, Mexico. Rev Biomed 2001; 12:224-30.

24.- Zavala Castro J, Acosta-Viana K, Bailón-Pacheco L, González-Robles A, Guzmán-Marín E, Rosales-Encina J. Kinetoplast DNA-binding protein profile in the epimastigotes form of Trypanosoma cruzi. Arch Med Res 2002; 33: 250-6.

25.- Zavala-Velázquez J, Barrera-Pérez M, Rodríguez-Félix ME, Guzmán-Marín E, Ruiz-Piña H. Infection by Trypanosoma cruzi in mammals in Yucatan, Mexico: a serological and parasitological study. Rev Inst Med Trop Sao Paulo 1996; 38: 289-92.

26.- Ruiz-Piña H, Cruz-Reyes A. The Opossum Didelphis virginiana as a synanthropic reservoir of Trypanosoma cruzi in Dzidzilche, Yucatan, Mexico. Mem Inst Oswaldo Cruz 2002. 97: 613-20. 